\title{
DESCRIPCIÓN DE LOS ESTILOS DE VIDA Y FACTORES DE RIESGO EN NIÑOS Y ADOLESCENTES DE TAMAULIPAS
}

\section{DESCRIPTION OF LIFESTYLES AND RISK FACTORS IN CHILDREN AND ADOLESCENTS FROM TAMAULIPAS}

\author{
Laura Elena Gaither Jiménez \\ Julio Herminio Pimienta Prieto
}

Doctora en Psicoterapia Gestalt (Universidad Gestalt de la Ciudad de México). Coordinadora del Centro de Investigación de la Subsecretaría de Educación Básica, Secretaría de Educación de Tamaulipas, México. Correo electrónico: [laura.gaither@gmail.com].

Doctorado en Educación «Diagnóstico, Medida y Evaluación de la Intervención Educativa» (Universidad Anáhuac México Norte, en convenio con la Universidad Complutense de Madrid). Subsecretario de Educación Básica en la Secretaría de Educación de Tamaulipas, México. Miembro del Sistema Nacional de Investigadores, Nivel I.

Correo electrónico: [juliopimienta1@gmail.com].

\section{RESUMEN}

La presente publicación forma parte de un estudio iniciado en 2012, teniendo como primer objetivo describir los estilos de vida y factores de riesgo detectados en niños y adolescentes, para la elaboración de un estudio diagnóstico que permitiera diseñar un plan 
de acción que atendiera los resultados obtenidos, utilizando como marco a los Consejos Escolares de Participación Social. Se trata de un estudio mixto, con una fase descriptiva y otra que emplea la investigación-acción-participativa. Se utilizó un muestreo estratificado en dos etapas - por escuelas y grupos-, en el que participaron 9,376 estudiantes de 131 primarias y 104 secundarias, de 34 municipios de los 43 que integran el estado de Tamaulipas, en México. Se usó un cuestionario propuesto por la Secretaría de Educación Pública con preguntas abiertas y cerradas. Los resultados muestran que la tercera parte de los estudiantes, emplean su tiempo libre viendo televisión y utilizando video-juegos; detectándose como factores de riesgo fundamentales, la incomprensión y escasa comunicación que percibe la inmensa mayoría, tanto por parte de los padres como de los docentes. Los resultados apuntan a la necesidad de políticas educativas estatales que atiendan la urgencia de involucrar a los padres de familia, mediante un programa de acompañamiento, y por otro lado, de intervención dentro de la institución, con un programa dirigido a la gestión escolar de una disciplina basada en acuerdos, respetando la dignidad y los derechos, y difundiendo las obligaciones de los padres.

Palabras clave: educación básica, adolescentes, estilos de vida, factores de riesgo, consejos escolares de participación social.

\section{ABSTRACT}

This publication is part of a study begun in 2012 with the first objective of describing the lifestyles and risk factors detected in children and adolescents, in order to prepare a diagnostic study that would allow the design of an action plan that would meet the results obtained, Using as a frame the School Social Participation Councils. This is a mixed study, with a descriptive phase and another using participatory action-research. Stratified sampling was used in two stages, by schools and groups, involving 9,376 students from 131 primary schools and 104 secondary schools from 34 municipalities of the 43 that make up the State of Tamaulipas in Mexico. We used a questionnaire proposed by the Ministry of Public Education with open and closed questions, 
and the results show that one-third of the students spend their free time watching television and using video games; Being detected as fundamental risk factors, incomprehension and poor communication, perceived by the vast majority, both by parents and teachers. The results point to the need for state educational policies that address the urgency of involving parents through an accompanying program and, on the other hand, intervention within the institution, with a program directed to the school management of a Discipline based on agreements, respecting the dignity and the rights, and spreading the obligations of the parents.

Key word: basic education, adolescents, lifestyles, risk factors, school councils for social participation.

\section{INTRODUCCIÓN}

El desarrollo humano implica interacción entre el individuo y su ambiente. El niño, desde su nacimiento, recibe la influencia del contexto que le facilitará o no, un proceso óptimo para su desarrollo biopsicosocioemocional.

La Organización Mundial de la Salud (OMS, 2010), define la adolescencia como «[...] el periodo de crecimiento y desarrollo humano que se produce después de la niñez y antes de la edad adulta, entre los 10 y los 19 años» (p. 1).

Según la UNICEF (2011):

Una reciente investigación neurocientífica muestra que, en los años de la adolescencia temprana, el cerebro experimenta un súbito desarrollo eléctrico y fisiológico. El número de células cerebrales pueden casi llegar a duplicarse en el curso de un año, en tanto las redes neuronales se reorganizan radicalmente, con las repercusiones consiguientes sobre la capacidad emocional, física y mental. El desarrollo físico y sexual, más precoz en las niñas - que entran en la pubertad unos 12 a 18 meses antes que los varones- se refleja en tendencias semejantes en el desarrollo del cerebro. 
El lóbulo frontal, la parte del cerebro que gobierna el razonamiento y la toma de decisiones, empieza a desarrollarse durante la adolescencia temprana. Debido a que este desarrollo comienza más tarde y toma más tiempo en los varones, la tendencia de éstos a actuar impulsivamente y a pensar de una manera acrítica, dura mucho más tiempo que en las niñas. Este fenómeno contribuye a la percepción generalizada de que las niñas maduran mucho antes que los varones (p. 6).

Es importante mencionar que no sólo influyen los cambios biológicos, la adolescencia es un concepto construido socialmente y posee connotaciones culturales. De acuerdo con Krauskopf (1994), es el periodo en que se produce con mayor intensidad la interacción entre las tendencias individuales, las adquisiciones psicosociales, las metas socialmente disponibles, las fortalezas y desventajas del entorno. El medio ambiente genera impacto en las costumbres, los valores, las actitudes, las emociones y en la percepción de sí mismo, en función de las características psicológicas y habilidades sociales.

La misma autora hace una diferenciación de las características personales, de acuerdo con la etapa.

Pre adolescencia (10-12 años):

- Preocupación por lo físico y emocional.

- Duelo por el cuerpo y por la relación infantil con los padres.

- Reestructuración del esquema e imagen corporal.

- Ajustes a emergentes cambios sexuales, físicos y fisiológicos.

- Estímulo de las nuevas posibilidades que abren estos cambios.

- Necesidad de compartir los problemas con los padres.

- Fluctuaciones del ánimo.

- Fuerte autoconciencia de necesidades.

- Capacidad de evaluar mayores méritos y debilidades.

- El juego mantiene su importancia. 
- La disciplina se acepta con exigencia de derechos y preocupación por la justicia.

- Afirmación a través de oposición.

- Curiosidad investigativa, interés por el debate.

- Relaciones grupales con el mismo sexo.

- Movimientos de regresión y avance en la exploración y abandono de la dependencia. (Krauskopf, 2011, pp. 2-3).

Por su parte, Salazar (2011) considera que los adolescentes:

Desarrollan un desafío a lo establecido, tienen deseos de independencia y la necesidad de elaborar un «yo». Es aquí donde lo experimentado por otros, cobra sentido. Mediante la comparación social o la interacción directa, por modelaje, las acciones de los demás sirven como indicadores para la organización situacional del sí-mismo. Por consiguiente, algunos jóvenes son más susceptibles, no discriminan y asumen modelos sociales que pueden causarles daño (p. 45).

El estilo de vida de los adolescentes se enfrenta a fluctuaciones derivadas de los mismos cambios sociales. De acuerdo con Bibeau (1985), desde una perspectiva integral, es necesario considerar los estilos de vida como parte de una dimensión colectiva y social, que comprende tres aspectos interrelacionados: material, social e ideológico.

Los estilos de vida saludables son procesos sociales: tradiciones, hábitos, conductas y comportamientos de los individuos y grupos de población que conllevan a la satisfacción de los requerimientos humanos para alcanzar el bienestar y la vida (Maya, 2001); la satisfacción de las necesidades humanas se despliega en tres contextos interrelacionados, en relación con uno mismo, con el grupo social y con el medio ambiente.

Cabe señalar, que los estilos de vida se interrelacionan con la presencia de factores de riesgo y/o de factores para el bienestar, y son parte de un proceso dinámico conformado con acciones o comportamientos individuales y acciones de naturaleza social. De acuerdo con 
Jessor: «El concepto de riesgo psicosocial hace referencia a toda la gama de desarrollo personal y adaptación social en la adolescencia» (p. 598).

Los adolescentes conforman una población vulnerable. Están expuestos al consumo de drogas, a la violencia, a las enfermedades de transmisión sexual o los embarazos no deseados, aspectos que impactan sobre el aprendizaje o permanencia en la escuela.

Los factores de riesgo y de protección pueden afectar a los niños durante diferentes etapas de sus vidas. En cada etapa ocurren riesgos que pueden modificarse a través de una intervención preventiva y que favorezca el desarrollo de conductas positivas o evite riesgos adicionales, como el fracaso académico.

Es posible hablar de factores individuales, sociales, culturales y escolares que, cuando confluyen, afectan gravemente el desarrollo sano y equilibrado de los adolescentes, e impiden que en el futuro se incorporen a la vida adulta con niveles aceptables de bienestar (UNICEF, 2000).

El Instituto Nacional sobre Abuso de Drogas (NIDA, 2017) de los Estados Unidos, menciona que:

- El riesgo de convertirse en un abusador de drogas involucra una relación entre el número y el tipo de los factores de riesgo (ejemplo: actitudes y comportamientos desviados), y los factores de protección (ejemplo: el apoyo de los padres).

- El impacto potencial de factores específicos de riesgo y de protección cambia con la edad. Por ejemplo, los factores de riesgo dentro de la familia adquieren un impacto mayor en un niño que en un joven, mientras que la asociación con compañeros que abusan de las drogas puede ser un factor de riesgo más importante para un adolescente.

- Una intervención temprana en los factores de riesgo (ejemplo: conducta agresiva y un auto-control deficiente), a menudo tienen un impacto mayor que una intervención tardía, al cambiar la trayectoria de la vida del niño, alejándole de los problemas y dirigiéndolo hacia conductas positivas. 
- Aunque los factores de riesgo y de protección pueden afectar a personas de todos los grupos, poseen un efecto diferente dependiendo de la edad, el sexo, la raza, la cultura y el ambiente de la persona (p. 2).

En el plano individual, los factores psicológicos se refieren a las características y los procesos internos (como el autoconcepto), que determinan la vulnerabilidad del adolescente frente a las influencias sociales. Algunos factores de riesgo estudiados predisponen al adolescente hacia el consumo de drogas, la violencia y el suicidio.

Entre los muchos cambios de comportamiento observados en los adolescentes, Johnson (2010) destaca tres que se analizan con frecuencia en diferentes culturas:

1) Aumento de la búsqueda de novedad; 2 ) aumento de la toma de riesgos, y 3) cambio de afiliación social hacia las interacciones entre pares. Esta tríada de cambios de comportamiento se observa no sólo en los seres humanos, sino en casi todos los mamíferos sociales. Aunque los comportamientos pueden conducir al peligro, confieren una ventaja evolutiva al motivar la separación de la comodidad y seguridad de la familia natal, lo que disminuye las posibilidades de endogamia. Los cambios de comportamiento también fomentan el desarrollo y la adquisición de habilidades independientes de supervivencia.

Con relación a los factores de riesgo familiares, es importante mencionar que una de las funciones de la familia es la socialización, por lo tanto, de acuerdo a la percepción de sí mismo y de su experiencia en la familia, es como el adolescente se relaciona con su medio. El primer cambio importante para los niños es entrar a la escuela y dejar la seguridad del entorno familiar.

Sobre las relaciones familiares NIDA (2017) considera que:

La compenetración familiar constituye el cimiento de la relación entre los padres y los hijos. Puede fortalecerse esta compenetración a través de un entrenamiento para mejorar la capacidad de apoyo de los padres a los hijos, la comunicación entre padres e hijos, y la participación de los padres con los hijos. El monitoreo y la supervisión de los padres son esenciales 
para prevenir el abuso de las drogas. Es factible mejorar estas habilidades con entrenamiento de cómo establecer reglas; técnicas para el monitoreo de actividades; elogio por conductas apropiadas; y disciplina moderada y consistente que posibilita respetar las reglas de la familia [...] (p. 3).

Cuando los alumnos avanzan de la escuela primaria a la secundaria, afrontan retos sociales, como por ejemplo, interactuar con un grupo mayor de compañeros. Es generalmente durante esta etapa, la adolescencia inicial, cuando los niños se enfrentan con el uso de drogas por primera vez, especialmente en forma experimental, como resultado de estar expuesto a modelos familiares, sociales o por evadir los problemas que presentan. A pesar de ello, la escuela sigue siendo un factor protector.

Al respecto, Medina-Mora, mencionada por la Comisión Nacional contra las Adicciones (2015), informa que:

[...] los estudios de escuelas han detectado el impacto de la creciente disponibilidad de cocaína en un incremento importante en el consumo; cuando se cambiaron las rutas de tránsito de esta droga, proveniente de la región andina hacia los Estados Unidos, dejó de pasar principalmente por el Caribe y se usaron las rutas de México, que ya operaban para el trasiego de heroína. Las encuestas de escuelas han documentado también la emergencia del crack y de las metanfetaminas, y han dado cuenta de los primeros logros de la política anti tabaco y de la persistencia del abuso de alcohol como principal problema de abuso (p. 16).

De acuerdo con la Encuesta de Estudiantes 2014, aplicada en México por el Instituto Nacional de Psiquiatría, con relación a la prevalencia de consumo de alcohol, se observa que a mayor edad mayor es el consumo. En los estudiantes de 12 años o menos, el 26.7\% consumió alcohol alguna vez en la vida, este porcentaje incrementa a $78.4 \%$ en los estudiantes de 17 años. Lo mismo ocurre con el consumo excesivo de alcohol y hacia los 12 años o menos, la prevalencia es de 4\%; para los estudiantes de 17 años esta prevalencia alcanza el $28.8 \%$ (CONADIC, 2015). 
Por otra parte, concurren factores de riesgo para la violencia escolar, sobre el particular Gabarda (2015) considera que:

Existe una serie de factores de riesgo o características (personales, familiares, escolares, sociales culturales, etc.) cuya presencia provoca que aumente la probabilidad de que se presenten fenómenos de violencia escolar (p. 6).

Asu vez, la misma autora hace mención de factores de riesgo del agresor:

- Ausencia de empatía: incapacidad para reconocer el estado emocional de otras personas.

- Baja autoestima: percepción negativa de sí mismo.

- Impulsividad: falta de control de los impulsos que lleva a actuar y decir las cosas sin pensar.

- Egocentrismo: exagerada exaltación de la propia personalidad, por lo que la persona se considera el centro de atención.

- Fracaso escolar: bajo rendimiento en los estudios, que puede llevar al absentismo y/o abandono escolar, consumo de alcohol y drogas.

- Trastornos psicopatológicos: trastornos de conducta, trastorno por déficit de atención con hiperactividad (TDH), trastorno negativista, desafiante y trastorno disocial. Antecedentes familiares de violencia. Entorno sociocultural bajo (Gabarda, 2015, p. 6).

Al analizar el papel de las víctimas, menciona como factores de riesgo los siguientes:

- Rasgos físicos o culturales distintos a la mayoría: minorías étnicas, raciales y culturales.

- Sufrir alguna discapacidad física o psíquica.

- Escasa comunicación familiar (Gabarda, 2015, p. 6). 
Algunas características como la inhibición o la dificultad para expresar emociones de los niños y adolescentes entorpecen su adaptación y los predisponen para desencadenar algunas alteraciones que interfieren con su aprendizaje. Al respecto, Taboada (1998) considera que:

[...] la inhibición conductual podría ser un factor de riesgo para el posterior desarrollo de trastornos de ansiedad en la infancia, mientras que la psicopatología de los padres, limitado a trastorno de angustia y agorafobia, podría conferir un cierto riesgo para la inhibición conductual en los niños (p. 49).

Ante esta realidad, es necesario el replanteamiento del Sistema Educativo, como indica Krauskopf:

Las transformaciones propias de la adolescencia, los efectos de la globalización, la modernización, las diferencias económicas, generan nuevas condiciones que confrontan la interacción y las metas que se propone el sistema escolar. La multiculturalidad está presente en todos lados. Se producen nuevas configuraciones y especificidades que tocan al trabajo y la educación (p. 8).

Los padres asumen un papel importante en el sistema educativo; sin embargo, algunos presentan resistencia para participar activamente, o su participación se limita a apoyar económicamente. La mayoría de los padres involucrados en las actividades escolares y extraescolares que apoyan la educación de sus hijos, son quienes menos necesitan acercamientos con la institución educativa, puesto que sus actuaciones constituyen factores de protección.

Algo favorable que ha surgido en años recientes, para propiciar la participación de los padres, es la estructura y normatividad existente en la Secretaría de Educación, misma que posibilita la integración de los Consejos Escolares de Participación Social (SET, 2010); pero también existen áreas de oportunidad en el deficiente seguimiento y la evaluación de los procesos institucionales relacionados directamente con los programas escolares. Algo que se percibe como amenaza es la ruptura 
que pudiera presentarse entre la escuela y la familia con interferencia en la participación comprometida de los padres y, por lo tanto, en el seguimiento y apoyo en el proceso educativo.

En el marco de la calidad educativa, pregonada en estos tiempos, es compromiso de la escuela asegurar el desarrollo armónico de los alumnos; por ello es esencial identificar los factores de riesgo que pueden interferir en el proceso y generar estrategias para disminuirlos, en forma coordinada con los integrantes de la comunidad educativa.

La coordinación estratégica de la comunidad educativa, se convierte en un área de oportunidad para disminuir los factores de riesgo y hacer, de las escuelas, comunidades saludables capaces de promover el desarrollo integral del adolescente, en función de la integración y operación de los Consejos Escolares de Participación Social (CEPS). Así se dará respuesta a las necesidades de los planteles educativos, teniendo como objetivo la formación de los niños y adolescentes. Por esto es indispensable identificar:

- ¿Qué características presenta el estilo de vida de los niños y adolescentes del estado?

- ¿Cuáles son los factores de riesgo presentes en este grupo?

En este contexto, se realizó un estudio descriptivo para elaborar un diagnóstico en las escuelas de nivel básico en el estado de Tamaulipas, México; para identificar las áreas y los factores de riesgo de los preadolescentes y adolescentes tempranos, y el nivel de riesgo en cada plantel, con el propósito de que cada escuela elabore un plan de acción que involucre a la comunidad educativa, y propicie el desarrollo de un programa integral de intervención.

Como objetivos específicos se consideraron:

1. Identificar los factores de riesgo en los preadolescentes y adolescentes tempranos, ubicados en escuelas primarias y secundarias del estado de Tamaulipas, y el nivel de riesgo de los planteles educativos.

2. Favorecer la participación de la comunidad educativa. 
3. Establecer un programa de intervención para disminuir los factores de riesgo, a través de los Consejos Escolares de Participación Social.

Los resultados del diagnóstico que presentamos en este momento -debido a que la investigación continúa- permiten identificar el nivel de riesgo de las escuelas participantes, las áreas de riesgo de los adolescentes y las situaciones que impactan en la vida de los preadolescentes y adolescentes, mismas que se presentan como una constante en los estudiantes de las diferentes regiones del estado, lo que orientará al impulso de estrategias integrales en las escuelas para favorecer su desarrollo personal y disminuir los factores de riesgo a los que están expuestos en su medioambiente social, escolar y familiar, tomando en consideración que la familia, junto con la escuela, son instituciones formativas.

\section{MÉTODO}

En una primera etapa, se empleó un diseño descriptivo para el que se ha utilizado un cuestionario con preguntas cerradas y abiertas, proporcionado por la Secretaría de Educación Pública, con la intención de determinar los factores de riesgo en escuelas primarias y secundarias del estado de Tamaulipas, en México. En una segunda etapa se ha trabajado mediante la perspectiva de la investigaciónacción-participativa, que se llevó a cabo en tres momentos: la elaboración de un manual para la operación de los Consejos Escolares de Participación Social (CEPS), elaboración de un diagnóstico, que fue utilizado finalmente para el diseño de un plan de acción para atender las necesidades detectadas en las escuelas participantes.

La muestra se seleccionó con base en el marco muestral de escuelas y estudiantes distribuidos en grupos y de los diversos municipios de la entidad, aportado por la Secretaría de Educación de Tamaulipas y correspondiente al ciclo escolar 2011-2012 (puesto que en ese momento se llevó a cabo la primera recolección de datos para esta investigación que aún continúa). El muestreo fue bietápico y estratificado, considerando la cantidad de estudiantes asignados a cada institución participante. 
El cuestionario fue aplicado a 9,376 estudiantes de $4^{\circ}, 5^{\circ}$ y $6^{\circ}$ grados de educación primaria y $1^{\circ}$ de secundaria. Fueron consideradas escuelas oficiales, particulares, federales y estatales, mismas que se estratificaron y distribuyeron de la siguiente manera: se aplicó en 131 primarias y 104 secundarias en 34 municipios y 40 localidades.

\section{RESULTADOS}

Atendiendo a la detección de las situaciones de riesgo de forma relevante y considerando su frecuencia, éstas son: divorcio de los padres; muerte, enfermedad o desaparición de algún familiar; además de violencia y conflictos familiares.

Los indicadores de riesgo relacionados con la prevalencia del consumo de drogas en las escuelas participantes que fueron reportados son: tensión emocional y corporal; baja autoestima; alimentación inadecuada; actividades de tiempo libre; deficiente comunicación con los padres y establecimiento de reglas en la familia.

A continuación los detallamos:

- Psicocorporal. Se identifica que la tercera parte de los alumnos encuestados, de nivel primaria y secundaria, presentan molestias físicas, como dolor de cabeza o de estómago, por lo menos una vez por semana, lo cual podría interferir con su desempeño académico. Asimismo, el 29\% de los alumnos de secundaria y el 35\% de los alumnos de primaria manifiestan ansiedad y dificultad para concentrarse.

- Autoconcepto. El 15\% de los alumnos de primaria y el 13\% de los alumnos de secundaria, no se sienten tomados en cuenta por padres o maestros. E1 35\% de los alumnos de primaria y el 32\% de los alumnos de secundaria no se sienten satisfechos con su apariencia. El $46 \%$ en primaria y $42 \%$ en secundaria reprime sus emociones cuando se sienten rechazados.

- Nutrición. La mayoría de los estudiantes de primaria (83\%) y secundaria $(82 \%)$, reportan tener una dieta balanceada y el $74 \%$ indica realizar las comidas en compañía de su familia. 
- Creatividad-economía. El 55\% de los alumnos reportaron tener su propia habitación y libertad para decorarla, mientras que un $45 \%$ reportó no tenerla.

- Consumo de drogas. El 9\% de los alumnos de primaria y secundaria señalaron que han consumido alcohol, varias veces, hasta llegar a la ebriedad, y el 17\% de la muestra, por nivel, reportan consumo de los padres y abuso ocasional. Con relación al consumo de tabaco, el $9 \%$ de los alumnos de primaria y el 6\% de los alumnos de secundaria manifestaron consumir más de 10 cigarrillos; mientras que el $20 \%$ en primaria y $19 \%$ en secundaria reportan consumo experimental. Por otra parte, el $7 \%$ de los alumnos en nivel primaria y $6 \%$ en secundaria señalan haber consumido una o más drogas ilícitas. El 7\% del total de la muestra ha consumido una o varios tipos de drogas ilegales.

- Tiempo libre. El 34\% de los alumnos de primaria y el 30\% de los alumnos de secundaria reportaron pasar la mayor parte de su tiempo viendo televisión, mientras que el $33 \%$ en primaria y $36 \%$ en secundaria indicaron que pasan muchas horas jugando en la computadora. La mayoría, $69 \%$ de primaria y $72 \%$ en secundaria, afirmó realizar ejercicios físicos.

- Expresión. La gran mayoría de los estudiantes, el $84 \%$ de secundaria y el $83 \%$ de primaria reportaron no sentirse escuchados, ni comprendidos por sus padres. La mayoría de los adolescentes, 70\% en primaria y $77 \%$ en secundaria, consideran que las reglas en su casa son muy claras.

Derivado del diagnóstico, escasas escuelas elaboraron un plan de acción para dar respuesta a sus necesidades, tomando como base a los factores identificados de riesgo. Por su parte, los programas de acción determinados, constituyen líneas que vagamente pudieran incidir en el fenómeno. Por ello, la Subsecretaría de Educación Básica, considera pertinente sugerir la utilización de dos programas estatales, uno dirigido al involucramiento de los padres de familia mediante el acompañamiento de la escuela y en el que la autoridad educativa estatal tenga un rol activo, apoyando la autonomía de gestión; pero, con la 
propuesta de los programas: «Convivencia familiar basada en acuerdos» y "Gestión de una disciplina escolar que respeta la dignidad y los derechos».

\section{DISCUSIÓN Y CONCLUSIONES}

Una tercera parte de los alumnos presentan, con frecuencia, tensión corporal y molestias físicas como dolor de cabeza o estómago, que se asocian con síntomas de ansiedad y pueden interferir en su desempeño académico. En esa misma proporción, presentan un bajo concepto de sí mismos y comunicación deficiente con los padres, que los lleva a percibir una actitud de incomprensión y rechazo en ellos, lo cual puede estar relacionado con las características de la edad, de acuerdo con lo señalado por Krauskopof (1999).

Con relación a la alimentación, la mayoría de los preadolescentes y adolescentes tempranos reportan hábitos adecuados de alimentación, en función de tener una dieta balanceada y de realizar las comidas en compañía de su familia. Asimismo, el 72\% de alumnos en secundaria y el $69 \%$ en primaria, llevan a cabo alguna actividad física.

Se identifica abuso de alcohol, consumo de tabaco y drogas en un porcentaje bajo de la población encuestada, ligeramente mayor en primaria que en secundaria. Con relación al alcohol, el 9\% presenta abuso y el $17 \%$ reporta consumo y abuso por parte de sus familiares. Al comparar los resultados que publica la Comisión Nacional contra las Adicciones (2015) con relación al consumo excesivo, se observa que éste se encuentra muy por encima de la media nacional $(4 \%)$ en primaria, siendo similar en secundaria (9\%). Asimismo, con relación al consumo de los familiares, la prevalencia en primarias se encuentra en el mismo nivel que la media nacional $(16.9 \%)$.

Al analizar el consumo de tabaco, se observa que el $9 \%$ en primaria y $6 \%$ en secundaria afirma consumir más de 10 cigarrillos por día; por otra parte, el $20 \%$ en primaria y $19 \%$ en secundaria reportan consumo experimental y es a esa población a la que deben dirigirse los programas de prevención selectiva. 
En otro sentido, el 7\% de la muestra indica haber utilizado drogas ilícitas. Entre las drogas ilícitas de mayor consumo se encuentran la marihuana, las inhalables y la cocaína; éstas son, de acuerdo con el reporte de CONADIC (2015), las drogas de mayor consumo identificadas durante la aplicación de la Encuesta Nacional. Es importante observar que reportaron consumo de helio en escuelas primarias de Río Bravo.

De acuerdo con el Consejo Nacional contra las Adicciones (2011), los programas de prevención para los estudiantes de educación básica y media superior deben enfocarse al mejoramiento del aprendizaje académico y socioemocional para tratar los factores de riesgo, como la agresión temprana, el fracaso escolar y la deserción, debiéndose considerar el desarrollo de habilidades de autocontrol, conciencia emocional, comunicación, solución de problemas y apoyo académico, con énfasis en las sustancias que está consumiendo.

Un tercio de la población dedica su tiempo libre a actividades sedentarias, como ver televisión o el uso de la computadora; sin embargo, la mayoría realiza alguna actividad física y esto puede actuar como un factor protector.

Los resultados del estudio permiten identificar que la gran mayoría de los adolescentes presentan dificultad en la comunicación con los padres, lo que impacta en su percepción y los hace sentirse incomprendidos, de acuerdo con Gabarda (2015) esto se convierte en factor de riesgo para la violencia escolar.

El 30\% de los preadolescentes y adolescentes tempranos ubicado en el nivel básico, manifiestan tensión corporal, molestia física, que puede ser ansiedad, de acuerdo con Tortella (2014); pero también pudiera relacionarse con un concepto devaluado de sí mismo. Resalta también que este porcentaje manifiesta que la mayor cantidad de su tiempo libre la dedican a ver televisión y videojuegos.

Por otra parte, la tensión corporal y el consumo de drogas como tabaco y alcohol están especialmente asociados con la ansiedad, porque generan una sensación inicial de placer, lo cual potencializa el riesgo al consumirse por los adolescentes; es por ello que se requiere abordar a los educandos en forma integral desde una 
consideración biopsicosocioemocional y desarrollar estrategias para canalizar la tensión y favorecer la expresión de emociones.

Los principales factores de riesgo están relacionados con las áreas de consumo de drogas, psicocorporal, autoconcepto y comunicación; deben considerarse estos factores en la planeación y el diseño de los programas estatales de intervención como políticas públicas locales.

La relación con los padres puede ser tanto un factor de riesgo, como un factor de protección. Factor de riesgo, cuando la comunicación es deficiente, cuando no se establecen normas o se implementan pero no se supervisa su cumplimiento y, sobre todo, cuando no se asocian a consecuencias lógicas por el rompimiento de las mismas. Por otra parte, los padres se convierten en importante modelo social y el abuso de alcohol, identificado por los hijos, puede impactar e incrementar la probabilidad de consumo y abuso por parte de éstos, como informa NIDA (2017).

Asimismo, la mayoría de los padres establecen reglas, mismas que son identificadas por los adolescentes; sin embargo, hay un $30 \%$ para el cual las reglas no son claras. A partir de ello se considera un área de oportunidad el acompañamiento de los padres en habilidades de comunicación y el manejo de la disciplina en el hogar.

Algunas limitaciones del estudio fueron el nivel de respuesta de los Consejos Escolares de Participación Social; con base al diagnóstico, sólo 357 (menos del 1\%) elaboraron un plan de acción para responder a las necesidades detectadas. Otra limitación se relaciona con aspectos logísticos de coordinación con la institución educativa para facilitar su participación y la supervisión de las personas que fungieron como enlaces municipales, ubicados en los Centros Regionales de Desarrollo Educativo. Para estudios posteriores es importante considerar una fase de sensibilización de la comunidad educativa y la supervisión estrecha de los enlaces en la comunidad con el propósito de facilitar la respuesta.

Se sugiere considerar los resultados para la promoción de políticas públicas que incidan en la disminución del riesgo y la potenciación de los factores de protección, a fin de asegurar el desarrollo integral de los estudiantes y, como consecuencia, elevar su bienestar. 
La participación de los adolescentes debe significar: expresar su opinión libremente, tener iniciativas y actuar en los procesos, para ello, la entidad deberá evaluar las políticas, los programas y servicios con el fin de garantizar que sean diseñados de acuerdo con sus necesidades e intereses.

Con relación al consumo de drogas, es importante fortalecer las políticas educativas e involucrar a los padres de familia y personal docente en las acciones integrales, además de considerar estrategias específicas para cada sustancia (SET, 2017).

\section{REFERENCIAS}

Alberich, T. (2007). Investigación - Acción Participativa y Mapas sociales. Madrid, España: Universidad de Jaén. Consultado en: http:// www.uji.es/bin/serveis/sasc/ext-uni/oferim/forma/ jorn/tall.pdf

Bailey, S. y Hubbard, R. (1991). Developmental Changes in Peer Factors and the Influence on Marijuana Initiation Among Secondary School Students. Journal of Youth and Adolescence, 20 (3), pp. 339-360.

Beltrán Llera, J. (2008). Psicología de la Educación. Madrid, España: Boixeareu Universitaria. España.

Castro, M. (1990). Indicadores de riesgo para el consumo problemático de drogas en jóvenes estudiantes. Aplicaciones en investigación y atención primaria dentro del plantel escolar. Salud Pública Méx 1990; Vol. 32(3), pp. 298-308.

Consejo Nacional contra las Adicciones (2000). Construye tu vida sin adicciones. Modelo de prevención. México: CONADIC. 
Centro Nacional para la Prevención y control de las Adicciones. Portal. Guía para educadores y profesionales de la salud. México: CENADIC. En: http://www.cenadic.salud.gob.mx/PDFS/ publicaciones/guiaed.pdf

Comisión Nacional contra las Adicciones. CONADIC. (2015). Encuesta Nacional de Consumo de Drogas en Estudiantes 2014. Reporte de alcohol. Distrito Federal, México: Secretaría de Salud.

Esnaola, I. (2008). El autoconcepto: perspectivas de investigación. Revista psicodidáctica. 13 (1), pp. 179-194.

Fondo de Naciones Unidas para la Infancia. UNICEF (2006). Índice de los Derechos de la Niñez Mexicana (IDNIII). México: UNICEF.

Gabarda, V. (2015). Bullying en el aula. Valencia, España: Universidad Internacional de Valencia.

Gaither, L., Pérez, R. y González, V. (2004). Encuesta sobre el consumo de drogas en estudiantes. Tamaulipas, México: Secretaría de Salud.

Jessor, R. (1991). Risk Behaviour in Adolescence: A Psychosocial Framework for Understanding and Action. Journal of Adolescence Health. En: http://www.colorado.edu/ibs/jessor/pubs/1991 Lessor JAH RiskBehaviorinAdolescence.pdf

Johnson, S., Blum, R. y Giedd, J. (2009). Adolescent Maturity and the Brain: The Promise and Pitfalls of Neuroscience Research in Adolescent. Health Policy, 45 (3), pp. 216-221.

Krauskopof, D. (1999). El desarrollo psicológico en la adolescencia: Las transformaciones en una época de cambios. Revista Adolescencia y salud. 1 (2).

Krauskopof, D. (2001). Los nuevos desafíos de la educación en el desarrollo juvenil. 
Krauskopof, D. (2011). El desarrollo en la adolescencia: las transformaciones psicosociales y los derechos en una época de cambios. Psicología.com

Maya, L. (2001). Los estilos de vida saludables: componente de la calidad de vida. Costa Rica: FUNLIBRE.

Martínez y Bracho (2007). Los Consejos de Participación Social en la Educación y el Programa Escuelas de Calidad. Jalapa, México: Centro de Investigaciones y Estudios Superiores en Antropología Social.

NIDA (2017). Cómo prevenir el uso de drogas en los niños adolescentes. USA: Instituto Nacional sobre el Abuso de Drogas. En: https:// www.drugabuse.gov/es/publicaciones/como-prevenir-eluso-de-drogas-en-los-ninos-y-los-adolescentes-segunda-edicion

Organización Mundial de la Salud [OMS] (2012). Desarrollo en la Adolescencia.

En: http://www.who.int/maternal child adolescent/topics/ adolescence/dev/es/

Salazar, M. (2011). Adolescencia y Trastornos Alimenticios: Influencia de los Modelos Televisivos. Costa Rica: Universidad de Costa Rica. En: http:/ /iip.ucr.ac.cr/sites/default/files/informes/informe4.pdf

Secretaría de Educación Pública [SEP] (2000). Reglamento de los Consejos de Participación social en la Educación. Tamaulipas, México: Secretaría de Educación de Tamaulipas.

Secretaría de Educación Pública [SEP] (2010). Acuerdo No .535 por el que se emiten los Lineamientos generales para la operación de los Consejos Escolares de Participación Social. México: Secretaría de Educación Pública.

Secretaría de Educación de Tamaulipas [SET]. (2017). Modelo Educativo de Tamaulipas (MET). Ciudad Victoria, Tamaulipas, México: Gobierno del Estado de Tamaulipas. 
Taboada, A., Ezpeleta, L. y De la Osa, N. (1998). Factores de riesgo de los trastornos de ansiedad en la infancia y la adolescencia: Una revisión. Apuntes de Psicología, 16 (1, 2), pp. 47-72.

Tortela-Feliu, M. (2014). Los trastornos de ansiedad en el DSM V. Medicina Psicosomática y Psiquiatría de Enlace. Revista Iberoamericana de Psicosomática (110).

Fondo de las Naciones Unidas para la Infancia [UNICEF] (2000). Adolescencia en América Latina y el Caribe: Orientaciones para la formulación de políticas públicas. En: http://www.unicef. org/lac/adolescencia en am latina.pdf

Fondo de las Naciones Unidas para la Infancia [UNICEF] (2011). Estado Mundial de la Infancia. La adolescencia: una época de oportunidades.

En: https://www.unicef.org/honduras/Estado mundial infancia 2011.pdf 